\title{
The flux phase problem on the ring
}

\author{
Fumihiko Nakano *
}

\begin{abstract}
We give a simple proof to derive the optimal flux which minimizes the ground state energy in one dimensional Hubbard model, provided the number of particles is even.
\end{abstract}

KEY WORDS: Hubbard model, flux phase problem.

\section{Introduction}

We consider the Hubbard model on the ring (i.e., one dimensional system with periodic boundary condition), where the magnetic flux is threaded through the ring. Our problem is to obtain the optimal flux which minimizes the ground state energy.

To be precise, we define the Hubbard Hamiltonian as follows:

$$
H:=\sum_{\sigma=\uparrow, \downarrow} \sum_{x=1}^{L} t_{x, x+1} c_{x+1, \sigma}^{\dagger} c_{x, \sigma}+(\text { h.c. })+\sum_{x=1}^{L} U_{x} n_{x, \uparrow} n_{x, \downarrow},
$$

where $L(L \geq 3)$ is the number of sites, the site $L+1$ is equivalent to the site $1, t_{x, x+1} \in \mathbf{C},\left|t_{x, x+1}\right| \neq 0, U_{x} \in \mathbf{R}, c_{x, \sigma}^{\dagger}$ (resp. $c_{x, \sigma}$ ) is the creation (resp. annihilation) operator which satisfies the canonical anticommutation relations, and $n_{x, \sigma}:=c_{x, \sigma}^{\dagger} c_{x, \sigma}$.

We write $t_{x, x+1}=\left|t_{x, x+1}\right| \exp \left[i \theta_{x, x+1}\right], \theta_{x, x+1} \in[0,2 \pi)$. Then, the flux which penetrates the ring is defined to be $\varphi:=\sum_{x=1}^{L} \theta_{x, x+1}$. The ground state energy $E$ (in some fixed number of particles $N_{e}$ ) can be regarded as a function of $\varphi$ (and hence we write $E=E(\varphi)$ ), because it does not depend on any choice

* present address: Department of Physics, Princeton University, Princeton, NJ08544, USA, On leave of absence from: Mathematical Institute, Tohoku University, Sendai, 980-77, Japan. 
of $\left\{\theta_{x, x+1}\right\}_{x=1}^{L}$ which satisfies $\sum_{x=1}^{L} \theta_{x, x+1}=\varphi$. Our aim is to obtain the flux $\varphi=\varphi_{\text {opt }}$ which attains $\min _{\varphi \in[0,2 \pi)} E(\varphi)$. We call $\varphi_{\text {opt }}$ as the optimal flux. In general, $\varphi_{\text {opt }}$ is not unique, and we will not discuss the uniqueness question in this paper.

There are some closely related problems in the literature (our problem is the same as mentioned in (3) below). (1) it appears in a theory of superconductivity [AM, W], (2) In the study of the persistent current [FMSWH, K, YF, FK], they discussed whether the response of the Hubbard ring to the external field is diamagnetic or paramagnetic, and the influence of the electron-electron interaction to this property, (3) In high dimensional lattice, the flux phase conjecture [HLRW] says that the optimal flux per plaquette is equal to the particle density per site. This implies that the diamagnetic feature, which widely holds in the one particle system, becomes opposite in high electron density regime. This conjecture was rigorously proved by Lieb $[\mathrm{L}]$ at half filling. Macris-Nachtergaele $[\mathrm{MN}]$ gave an improved proof of $[\mathrm{L}]$.

As for the rigorous study of the Hubbard ring (of even length), Lieb-Loss [LL] considered free electron case $\left(U_{x} \equiv 0\right)$ at half filling, and computed $\varphi_{\text {opt }}$ in general situation so that translation invariance is not assumed. They also considered what have more complicated geometry such as tree of ring, ladder, etc. Lieb-Nachtergaele [LN] computed $\varphi_{\text {opt }}$ also at half filling when $U_{x} \equiv U$ is any constant. In this paper, we obtain $\varphi_{\text {opt }}$ when $U_{x}$ and $L$ are arbitrary, while $N_{e}$ is even. Due to the hole-particle symmetry, it suffices to consider $N_{e} \leq L$.

\section{Theorem}

Let $N_{e}(\leq L)$ be even.

(1) Assume $U_{x}<+\infty$ for all $x$. $E(\varphi)$ is minimized if $\varphi \equiv\left(N_{e} / 2+1\right) \pi(\bmod$ $2 \pi$ ) (resp. $\varphi \equiv N_{e} \pi / 2$ ) when $L$ is even (resp. $L$ is odd).

(2) When $U_{x}=\infty$ for all $x, E(\varphi)$ is minimized if $\varphi=0, \pi$.

\section{Remarks.}

(1) We can derive the optimal flux in $S^{z} \neq 0$ subspaces.

(a) $U_{x}<+\infty$ : the optimal flux takes 0 and $\pi$ alternatively as $S^{z}$ varies. For instance, when $N_{e}=4 n$, and $L$ is even, then $\varphi_{\text {opt }}=\pi\left(S^{z}=0,2,4, \cdots\right)$, and $\varphi_{\text {opt }}=0\left(S^{z}=1,3,5, \cdots\right)$.

(b) $U_{x} \equiv \infty$ : let $m:=N_{\uparrow} / N_{\downarrow}\left(N_{\uparrow}\right.$ (resp. $\left.N_{\downarrow}\right)$ is the number of up (resp. down) spins). We suppose $N_{\uparrow} \geq N_{\downarrow}$ here. When $m \notin \mathbf{N}, \varphi_{\text {opt }}=2 k \pi / N_{e}$, 
$k \in \mathbf{Z}$ (in this case, particles can also be regarded as hard core bosons). When $m \in \mathbf{N}, \varphi_{o p t}=2 k \pi /(m+1)-\left(N_{e}-1\right) \pi($ if $(m+1) L$ is even $)$, and $\varphi_{\text {opt }}=$ $(2 k-1) \pi /(m+1)-\left(N_{e}-1\right) \pi$ (if $(m+1) L$ is odd), $k \in \mathbf{Z}$.

(2) When $U_{x} \equiv+\infty$, the proof of Theorem tells us that the ground state energy is periodic w.r.t. $\varphi$ with period $\pi$ (when $S^{z}=0$ ), and period $2 \pi / N_{e}$ (when $m \notin \mathbf{Z}$ ). This fact and its implications are discussed by Kusmartsev and Yu-Fowler[K, YF].

(3) When $N_{e}$ is odd and $U_{x} \equiv+\infty$, we can still derive the optimal flux, and the result is the same as stated in Remark (1).

On the other hand, when $U_{x}<+\infty$, and $N_{e}=L$ (half-filling), we believe $\varphi_{\text {opt }}=\pi / 2,3 \pi / 2$ as some examples imply (e.g., take $t_{x, x+1}$ : constant and $\left.U_{x} \equiv 0\right)$. However, in general cases, $\varphi_{\text {opt }}$ could be different depending on the value of $U_{x}$. For example, let $L=4, N_{e}=3$, and $t_{x, x+1} \equiv t$. When $U_{x} \equiv 0$, $E(\varphi)$ is minimized if and only if $\varphi= \pm 4 \arcsin (1 / \sqrt{5})$, while in case of $U_{x} \equiv+\infty$, $E(\varphi)$ is minimized if and only if $\varphi=0,2 \pi / 3,4 \pi / 3$.

(4) $S U(2)$ invariance as well as translation invariance is not necessary to prove Theorem. We can let $t_{x, x+1}=t_{x, x+1}^{\sigma}(\sigma=\uparrow, \downarrow)$ depend also on spin variable. In this case, our theorem mentions the optimal flux in the $S^{z}=0$ subspace only. Besides, our Hamiltonian can include the one body potential term as well.

(5) The argument in the proof, together with that in [LM] yields the ground state is unique and has spin zero: $S=0$, provided the flux $\varphi$ takes the value as stated in Theorem. Moreover, if we let $E(S)$ denote the ground state energy in spin $S$ subspace, then we have $E(S)<E(S+2)$. It becomes equality when $U_{x} \equiv \infty, \varphi=\pi$ (resp. $\left.\varphi=0\right)$, and $L$ : even (resp. $L$ : odd).

(6) In general, the spin of the ground state is sensitive to the flux. For example, let $L$ be even, $N_{e}=4 n+2, t_{x, x+1} \equiv t$, and $U_{x} \equiv \infty$. Then, one can show that, (a) when $\varphi=0$, there is a singlet ground state, but no ferromagnetic ones. (b) when $\varphi=\pi$, there is a ferromagnetic ground state.

(7) When $U_{x}=\infty$, not for all $x$, the argument of the proof says the following: if $\sharp\left\{x: U_{x}=\infty\right\} \leq L-N_{e} / 2$, then the result is the same as in Theorem (1). Otherwise, the result is the same as in Theorem (2).

(8) When $U_{x} \equiv 0$, and $t_{x, x+1} \equiv t, E(\varphi)$ is maximized if and only if $\varphi \equiv$ $N_{e} \pi / 2$ (resp. $\left.\varphi \equiv\left(N_{e} / 2+1\right) \pi\right)$, if $L$ is even (resp. $L$ is odd), which should be compared with the fact that $E(0)=E(\pi)$ when $U_{x} \equiv \infty$ (Theorem (2)). 
(9) When we let $L$ large, $|E(0)-E(\varphi)|$ will behave as $O(1 / L)[\mathrm{LN}]$.

In section 2, we give the proof of Theorem, which is very simple. Our problem is reduced to consider an one-particle Hamiltonian $\mathcal{H}(\varphi)$ on the graph $G$ which is composed of the basis of $N_{e}$-fermion Hilbert space. Theorem follows from obtaining the optimal flux of $\mathcal{H}(\varphi)$ on $G$, by the usual diamagnetic inequality argument.

\section{Proof of Theorem}

At first, we consider the case in which $L$ is even and $N_{e}=4 n$. Due to the $S U(2)$ invariance, it is sufficient to work on $S^{z}=0$ subspace (i.e., $N_{\uparrow}=N_{\downarrow}=$ $2 n)$. We fix the basis of the Hilbert space of $N_{e}$-fermions:

$$
\begin{aligned}
\mathcal{B}:=\left\{c_{x_{1}, \sigma_{1}}^{\dagger} c_{x_{2}, \sigma_{2}}^{\dagger} \cdots c_{x_{N_{e}}, \sigma_{N_{e}}}^{\dagger} \mid \operatorname{vac}>:\right. & \\
& \left.x_{1} \leq x_{2} \leq \cdots \leq x_{N_{e}}, \sigma_{i}=\uparrow, \downarrow, i=1, \cdots, N_{e}\right\},
\end{aligned}
$$

that is, to arrange particles in increasing order w.r.t. the space coordinates. Our problem is equivalent to consider the one-particle Hamiltonian $\mathcal{H}(\varphi)$ on the graph $G$ whose sites are composed of $\mathcal{B}$.

$$
(\mathcal{H}(\varphi) u)(x):=\sum_{y \in \mathcal{B}} s_{x y}(\varphi) u(y)
$$

where $s_{x y}(\varphi):=<x|H| y>, x, y \in \mathcal{B}$. Two sites $x, y \in G$ are connected by a bond if and only if $s_{x y}(\varphi) \neq 0$ (we note $\left|s_{x y}(\varphi)\right|$ does not depend on $\varphi$ ). For given $\varphi \in[0,2 \pi)$, we fix some $\left\{\theta_{x, x+1}\right\}_{x=1}^{L}$ such that $\sum_{x=1}^{L} \theta_{x, x+1}=\varphi$, and thus we suppose $s_{x y}(\varphi)$, and hence $\mathcal{H}(\varphi)$, is determined by $\varphi$. Then, it is not hard to show that: (1) every circuit in $G$ has even length (because $L$ is even), (2) the fluxes in these circuits are always integer multiple of $\psi:=\varphi+2 n \pi+(4 n-1) \pi$. In fact, let $\mathcal{C}$ be the set of circuits in $G$ which have minimal length. Every elements of $\mathcal{C}$ is given by fixing all particles which have down (resp. up) spins and moving each up (resp. down) spins all together until each spins come to next spin. To make it clear, we write down an element of $\mathcal{C}$ when $L=N_{e}=4$ :

$$
\begin{array}{ccc}
c_{1, \uparrow}^{\dagger} c_{2, \downarrow}^{\dagger} c_{3, \uparrow}^{\dagger} c_{4, \downarrow}^{\dagger} \mid \operatorname{vac}> & \leftarrow c_{2, \downarrow}^{\dagger} c_{3, \uparrow}^{\dagger} c_{4, \uparrow}^{\dagger} c_{4, \downarrow}^{\dagger} \mid \operatorname{vac}> \\
\downarrow & \uparrow \\
c_{2, \uparrow}^{\dagger} c_{2, \downarrow}^{\dagger} c_{3, \uparrow}^{\dagger} c_{4, \downarrow}^{\dagger} \mid \operatorname{vac}> & \rightarrow c_{2, \uparrow}^{\dagger} c_{2, \downarrow}^{\dagger} c_{4, \uparrow}^{\dagger} c_{4, \downarrow}^{\dagger} \mid \operatorname{vac}>
\end{array}
$$


The second term $2 n \pi$ in the definition of $\psi$ comes from the fact that up spins jump down spins $2 n$ times on the above process, and each jump causes to put $(-1)$ on the corresponding $s_{x y}(\varphi)$. The third term $(4 n-1) \pi$ in the definition of $\psi$ comes from the fact that the $2 n$-th up spin jumps all the other $(4 n-1)$ particles when it moves from the site $L$ to the site 1 , because we set the basis such that particles are arranged in increasing order.

On the other hand, because of the inequality: $\sum_{x, y \in \mathcal{B}} s_{x y}(\varphi) \overline{u(x)} u(y) \geq$ $-\sum_{x, y \in \mathcal{B}}\left|s_{x y}(\varphi)\right||u(x)||u(y)|$, we know that the ground state energy is minimized when all off-diagonal elements $s_{x y}(\varphi), x \neq y$, are non-positive. Let $\left(\mathcal{H}_{-} u\right)(x):=-\sum_{y \in \mathcal{B}}\left|s_{x y}(\varphi)\right| u(y)$. When $\psi \equiv 0(\bmod 2 \pi), \mathcal{H}(\varphi)$ is unitarily equivalent to $\mathcal{H}_{-}$, because the fluxes of all circuits in $G$ are all the same [LL, Lemma 2.1$]. \psi \equiv 0(\bmod 2 \pi)$ yields $\varphi \equiv \pi(\bmod 2 \pi)$. This concludes the proof when $L$ is even and $N_{e}=4 n$.

When $L$ is even and $N_{e}=4 n+2$, the only thing we have to do is to replace $\psi$ in the above argument by $\psi^{\prime}:=\varphi+(2 n+1) \pi+(4 n+1) \pi$. When $L$ is odd, then $\psi$ (or $\psi^{\prime}$ in case of $\left.N_{e}=4 n+2\right)$, should satisfy $\psi \equiv \pi(\bmod 2 \pi)$ to have optimal flux, because the minimal length of the circuits in $G$ is odd, and so, the flux of $\mathcal{H}_{-}$on every elements of $\mathcal{C}$ is $\pi$. When $U_{x} \equiv \infty$, the minimal length of circuits in $G$ is $2 L$, whose flux is $2 \varphi+2\left(N_{e}-1\right) \pi \equiv 2 \varphi(\bmod 2 \pi)$.

Remarks

(1) As an alternative proof, one can compute the partition function $P(\varphi):=$ $\operatorname{Tr}[\exp (-\beta H)]$ by using the path integral representation $[\mathrm{AL}]$, and show that $P(\varphi)$ is maximized if $\varphi$ takes the value stated in Theorem. This approach has been done by [GMMU], where they derived the optimal flux in the FalicovKimball model.

(2) When the number of electrons is odd, the fluxes of elements of $\mathcal{C}$ are different from each other, depending on which spins move in the circuit. For example, let $L=N_{e}=2 n+1, N_{\uparrow}=n$, and $N_{\downarrow}=n+1$. By the hole-particle transformation only for down spins, we can suppose $N_{\uparrow}=N_{\downarrow}=n$, but now the flux of down spins is $\pi-\varphi$ (this situation is similar to that discussed in [FK]).

Our supposition is the following: the "contribution" to the ground state energy from $\mathcal{C}$ would cancel each other, and an important contribution would come from those circuits where up spins and down spins move together in the opposite direction which has flux $\varphi-(\pi-\varphi)=2 \varphi-\pi$, and has length $2 n$ in 
$G$ (the meaning of "contribution" could be clear if we consider $\operatorname{Tr}[\exp (-\beta H)]$ instead of the ground state energy). $2 \varphi-\pi \equiv 0$ would give the minimizing energy. However, this supposition would not be easy to prove.

(3) The proof above relies on the special nature of the ring geometry: there is always fixed number of particles on only one loop, so that all circuits on the graph $G$ favor the same flux 0 or $\pi$, depending on cases. However, on more complicated systems such as two dimensional lattice, the graph $G$ has so many different circuits which favor different fluxes so that our argument does not work even if $U_{x} \equiv \infty$, except the Nagaoka-case $\left(N_{e}=|\Lambda|-1, U_{x} \equiv \infty[\mathrm{N}, \mathrm{T}]\right)$, where the optimal flux is zero everywhere.

\section{Conclusion}

In this paper, we derived the optimal flux $\varphi_{\text {opt }}$ in the Hubbard model on the ring. Our result is true in general situation so that the translation invariance is not necessary to assume, except the number of particles must be even. In this section, we briefly discuss the physical interpretation of our result.

The result (1) of our theorem is consistent with that of [FMSWH], where it is shown that, at half-filling, the current response of the ground state is paramagnetic (resp. diamagnetic) when $N_{e}=4 n$ (resp. $4 n+2$ ) by numerical computation. However, these are not equivalent, especially when $N_{e}=4 n$. In fact, [FMSWH] showed, when $L=6, N_{e}=4$, and $U_{x}>0$, the ground state is diamagnetic (this also implies why it is not easy to seek $\varphi$ which maximizes $E(\varphi)$ ). Therefore, our contribution may be that there would be no effects of spatial disorder.

The result (2) of our theorem and Remark (2) after that is already found and discussed by [K, YF]. However, our proof gives a different picture: the graph $G$ consists of rings of larger lengths, for $U=\infty$ prohibits the exchange of particles.

Finally, our argument gives a ring version of the Lieb-Mattis theorem [LM] when $\varphi=\varphi_{\text {opt }}$ (Remark (5) after Theorem).

\section{Acknowledgement}

The author would like to thank professor E. H. Lieb for pointing out Remark (4)(5). The author is partially supported by the Japan Society for the Promotion of Science. 


\section{References}

[AL] Aizenman, M., and Lieb, E. H., Magnetic Properties of Some Itinerant-Electron Systems at T>0, Phys. Rev. Lett. 65(1990), 14701473.

[AM] Affleck, I., and Marston, J. B., Large n-limit of the HeisenbergHubbard model: Implications for high- $T_{c}$ superconductors, Phys. Rev. B37(1988), 3774-3777.

[FK] Fujimoto, S., and Kawakami, N., Persistent currents in mesoscopic Hubbard rings with spin-orbit interaction, Phys. Rev. B48(1993), 17406-17412.

[FMSWH] Fye, R. M., Martins, M. J., Scalapino, D. J., Wagner, J., and Hanke, W., Drude Weight, optical conductivity, and flux properties of onedimensional Hubbard rings, Phys. Rev. B44(1991), 6909-6915.

[GMMU] Gruber, C., Macris, N., Messager, A., and Ueltschi, D., Ground States and Flux Configurations of the Two-Dimensional FalicovKimball Model, J. Stat. Phys. 86(1997), 57-108.

[HLRW] Hasegawa, Y., Lederer, P., Rice, T. M., and Wiegmann, P. B., Theory of Electronic Diamagnetism in Two-Dimensional Lattices, Phys. Rev. Lett. 63(1989), 907-910.

[K] Kusmartsev, F. V., Magnetic resonance on a ring of aromatic molecules, J. Phys. Condensed Matter 3(1991), 3199-3204.

[L] Lieb, E. H., Flux Phase of the Half-Filled Band, Phys. Rev. Lett. 73(1994), 2158-2161.

[LL] Lieb, E. H., and Loss, M., Fluxes, Laplacians and Kasteleyn's theorem, Duke Math. J. 71(1993), 337-363.

[LM] Lieb, E. H., and Mattis, D. C., Theory of ferromagnetism and the ordering of electronic levels, Phys. Rev. 125(1962), 164-172.

[LN] Lieb, E. H., and Nachtergaele, B., Stability of the Peierls instability for ring-shaped molecules, Phys. Rev. B51(1995), 4777-4791. 
[MN] Macris, N., and Nachtergaele, B., On the flux phase conjecture at half-filling: an improved proof, J. Stat. Phys. 85(1996), 745-761.

[N] Nagaoka, Y., Ferromagnetism in a narrow, almost half-filled s band, Phys. Rev. 147(1966), 392-405.

[T] Thouless, D. J., Exchange in Solid ${ }^{3}$ He and the Heisenberg Hamiltonian, Proc. Phys. Soc. (London), 86(1965), 893-904.

[W] Wiegmann, P. B., Towards A Gauge Theory of Strongly Correlated Electronic Systems, Physica C 153-155(1988), 103-108.

[YF] Yu, F., and Fowler, M., Persistent current of a Hubbard ring threaded with a magnetic flux, Phys. Rev. B45(1992), 11795-11804. 